\title{
Identificación de los atributos del servicio en un centro de transformación de información digital y contable
}

\section{Identification of the attributes of the service in a digital and accounting information processing center}

GÓMEZ-MÁRQUEZ, Montserrat†*, RUIZ-HERNÁNDEZ, Ana Lilia, GONZÁLEZ-SÓBAL, Martín y ROSAS-BALTAZAR, Miguel Eduardo

Instituto Tecnológico Superior de Huatusco. Avenida 25 poniente, No. 100 Col. Reserva Territorial, Huatusco, Ver, C.P. 94100

Instituto Tecnológico Superior de Xalapa.

ID $1^{\text {er }}$ Autor: Montserrat, Gómez-Márquez / ORC ID: 0000-0002-2901-1745, Researcher ID Thomson: S-8535-2018, CVU CONACYT ID: 999026

ID $1^{\text {er }}$ Coautor: Ana Lilia, Ruiz- Hernández / ORC ID: 0000-0003-0945-6154, Researcher ID Thomson: W-8106-2019, CVU CONACYT ID: 346460

ID $2^{\text {do }}$ Coautor: Martín González -Sóbal / ORC ID: 0000-0003-0038-8319, Researcher ID Thomson: S-7631-2018, CVU CONACYT ID: 463431

ID $3^{\text {er }}$ Coautor: Miguel Eduardo Rosas Baltazar / ORC ID: 0000-0002-5821-0065, Researcher ID Thomson: W-6525-2019, CVU CONACYT ID: 218942

DOI: 10.35429/JIT.2019.21.6.9.19

Recibido: 10 de Agosto, 2019; Aceptado 30 de Diciembre, 2019

\section{Resumen}

Un Centro de Transformación de Información Digital y Contable (CTICD) es un espacio físico que cuenta con servicios de: préstamo de equipos de cómputo, accesorios y/o herramientas digitales (software, soporte técnico etc.). Cada CTICD tiene atributos tangibles e intangibles en el servicio que los distingue y los hace atractivos para el usuario. El objetivo del trabajo fue identificar los atributos que el usuario de un CTICD considera de mayor influencia para su existencia. El instrumento de recolección de datos se validó a través de la correlación de Pearson y se calculó la dependencia entre los atributos tangibles e intangibles de un CTICD. El resultado obtenido se generó con la prueba de independencia estadística chi-cuadrada. Se graficó chi-cuadrada teórica de un par de variables. La contribución de la presente investigación es la identificación e importancia que tienen los atributos intangibles en la percepción y diferenciación del servicio de un CTICD para los usuarios, resaltando la parte humanística a través de la atención a los mismos, anteponiéndola a cualquier atributo tangible.

Centro de Transformación de Información Digital y Contable, Atributos tangibles y atributos intangibles

\begin{abstract}
A Digital and Accounting Information Transformation Center (CTICD) is a physical space that has services such as: loan of computer equipment, accessories and / or digital tools (software, technical support, etc.). Each CTICD has tangible and intangible attributes in the service that distinguishes them and makes them attractive to the user. The objective of the work was to identify the attributes that the user of a CTICD considers of greater influence for its existence. The data collection instrument was validated through Pearson's correlation and the dependence between the tangible and intangible attributes of a CTICD was calculated. The result obtained was generated with the chi-square statistical independence test. Theoretical chi-square of a couple of variables was plotted. The contribution of this research is the identification and importance of intangible attributes in the perception and differentiation of the service of a CTICD for users, highlighting the humanistic part through attention to them, putting it before any tangible attribute.
\end{abstract}

Center for Transformation of Digital and Accounting Information, Tangible attributes and intangible attribute

Citación: GÓMEZ-MÁRQUEZ, Montserrat, RUIZ-HERNÁNDEZ, Ana Lilia, GONZÁLEZ-SÓBAL, Martín y ROSASBALTAZAR, Miguel Eduardo. Identificación de los atributos del servicio en un centro de transformación de información digital y contable. Revista de Tecnologías de la Información. 2019. 6-21: 9-19

\footnotetext{
$\dagger$ Investigador contribuyendo como primer autor.
} 


\section{Introducción}

Para exponer el tema de esta investigación es necesario explicar primero el origen de las computadoras, las cuales dan pauta a la existencia de los centros del cómputo. Según Gordon (2004), oficialmente la primera generación de computadoras (1945-1956) está constituida por equipos desarrollados en la época de los cincuenta con bulbos. Para 1951 destaca, la construcción de la computadora UNIVAC 1 , primera computadora comercial, durante esta época solo existían 20 máquinas en Estados Unidos y una gran saturación de datos que procesar, eran equipos costosos y solo tenían aplicaciones en el área científica y militar además utilizaban tarjetas perforadas.

Durante esta época se construyeron computadoras electrónicas que fueron usadas cada vez más para el manejo de datos en los negocios y en los procesos industriales. La segunda generación de computadoras (19561963) la marco el uso del transistor, dispositivo electrónico que generaba menos calor y consumía menos energía que los bulbos. Aquí aparecen grandes compañías como IBM y los primeros lenguajes de alto nivel produciéndose computadoras más pequeñas y rápidas con menores necesidades de ventilación, un evento importante fue la aparición de la Whirlwind I de Massachusetts Institute of Technology que fue diseñada para cálculos científicos en gran escala y para simuladores de vuelo en tiempo real, empleando núcleos electroestáticos y magnéticos (Ralston y Edwin, 1993).

Más adelante durante la tercera generación (1964-1971) se incorporan los circuitos integrados a los equipos de cómputo, inicia introduciendo la multiprogramación y el multiproceso, aparecen los lenguajes de alto nivel como Cobol y Fortran; IBM lanza a al mercado el modelo 360. La cuarta generación (1971 al presente), a partir de 1968 se inicia el concepto actual de las computadoras con la creación del microprocesador de Intel que incluía todos los componentes en un microprocesador y el cual al fabricarse en serie ha permitido una disminución de los costos. Durante la quinta generación de computadoras se incorporan tecnologías avanzadas relacionadas a la aplicación de inteligencia artificial con múltiples microprocesadores, se destaca una mayor de capacidad en paralelo de múltiples microprocesadores (1984-1990).
En 1990 surge una nueva generación explotando la de redes de cómputo. En México y en América latina la primera computadora que se instaló fue en la UNAM en julio de 1958 un cerebro electrónico IBM -650 con dimensiones físicas considerables pero con una capacidad de cálculo y almacenamiento inferiores a los de las computadoras actuales, el acceso a la máquina quedó restringido a una pequeña elite de investigadores sin embargo generó un fuerte interés e impulsó su estudio entre los jóvenes alumnos de las carreras de física y matemáticas que más tarde formarían la primera generación de computólogos mexicanos.

En 1960 el centro de cálculo de la UNAM adquirió un equipo Bendix G-15 el cual amplió en algunos meses el circulo de usuarios al integrar a estudiantes y profesores de ingeniería química así como alumnos y docentes del Instituto Politécnico Nacional, más a delante se adquirió un equipo más sofisticado tanto en la UNAM como en el IPN, este último creo el centro nacional de cálculo CENAC donde se formó un nutrido grupo de estudiantes de la ESIME que posteriormente en compañía de los egresados de la UNAM habrían de dirigir los principales centros de cómputo gubernamentales y de la industria privada (Carrillo, 1996).

De esta manera retomamos los inicios de los laboratorios de cómputo motivo de este trabajo, según Usman, Mehmood, y Katib, (2018) un centro de computación de alto desempeño es una colección de computadoras denominadas nodos los cuales se encuentran interconectados a través de una red de alta velocidad establecidos en una arquitectura específica para la ejecución de programas y procesamiento de información en paralelo. Una de las principales motivaciones en el uso de Centros de Computación de alto desempeño, es la paralelización de algoritmos para la obtención del mejor desempeño, reduciendo tiempo de cómputo haciendo un uso eficiente de los recursos en el procesamiento de grandes volúmenes de información.

Como podemos observar por los constantes avances tecnológicos que impulsan la dinámica de la sociedad actual, se imponen a las instituciones educacionales, en particular a las de educación superior. 
La necesidad de realizar transformaciones en sus procesos formativos, de modo que estos respondan a la formación de futuros profesionales que estén preparados para dar una respuesta adecuada a las actuales circunstancias de los entornos sociales donde se desenvuelven (Quiroz, 2019), es aquí donde entran los laboratorios de cómputo por la introducción de las tecnologías de la información y la comunicación de base informática y en especial Internet, que permiten la coordinación de tareas y la gestión competitiva, suministrando una combinación sin precedentes de flexibilidad y eficacia en la realización de las actividades, de toma de decisiones coordinadas y ejecución descentralizada, de expresión individualizada y comunicación global y horizontal, lo que permite el desarrollo de una forma organizativa superior de la actividad humana, (Castells, 2001).

Es importante hacer notar que "La gestión de la información académica en los centros de estudios sean privados o estatales requiere el uso de mecanismos que aseguren un manejo eficiente y contribuyan a incrementar la calidad de los servicios que se brindan a los alumnos" (Onofre y Catelin, 2014). Dicho de otra manera, los centros de cómputo escolares son lugares clave para alcanzar los objetivos institucionales, aunque en definitiva "Los departamentos de informática de cualquier empresa son importantes pero la tecnología por sí sola no resuelve los problemas, es necesarios que alguien cuide de ella y sirva de enlace entre ella y el usuario al cual sirve, pero no es posible eliminar el factor humano de este enlace (Casas de la Torre y Suarez, 2018).

En la actualidad todo se enfoca como prioridad al desarrollo del aspecto tecnológico parte tangible en un laboratorio de cómputo, sin embargo, la contribución directa de este trabajo de investigación es identificar la relación entre el servicio que se ofrece en un laboratorio de cómputo escolar por diversos atributos intangibles con los que cuenta y la tecnología de vanguardia que presenta.
"Dado que los servicios consisten en actuaciones y no en objetos como los productos, en su mayoría no se pueden probar. La calidad se produce durante la prestación del servicio, generalmente en una interacción entre el cliente y el personal de contacto de la organización, por lo que la calidad del servicio depende en gran medida del desempeño de los empleados" (Ornelas, Montelongo y Nájera, p. 40, 2010).

A su vez "la percepción de la calidad de un servicio, resulta ser similar a la actitud hacia un servicio, ya que se realiza una apreciación de forma global. Esta apreciación es individual y delimita las preferencias del usuario. Visto desde este punto de vista la satisfacción del cliente de manera general se 'puede agrupar en:

1.- El rendimiento percibido: se refiere al desempeño (en cuanto a la entrega de valor) que el cliente considera haber obtenido luego de adquirir un producto o servicio. Dicho de otro modo, es el "resultado" que el cliente "percibe" que obtuvo en el producto o servicio que adquirió.

2.- Las expectativas: las expectativas son las esperanzas que los clientes tienen por conseguir algo. Las expectativas de los clientes se producen por el efecto de una o más de estas cuatro situaciones: 1.- Promesa que hace la misma empresa acerca de los beneficios que brinda el producto o servicio. 2.- Experiencia de compra anterior. 3.- Opiniones de amistades, familiares, conocidos y líderes de opinión. 4.Promesas que ofrecen los competidores. (Ornelas, C. et al, 2010).

Zeithaml, Parasuraman y Berry en su libro titulado "Calidad total en la gestión de servicios" publicado en 1993 propone un instrumento llamado Servqual, que mide la calidad del servicio, de ahí surgen otros autores que manejan los atributos de la calidad mediante dimensiones. "Existen 5 dimensiones de la calidad del servicio: tangibilidad, fiabilidad, capacidad de respuesta, seguridad y empatía, definidos como sigue:

Tangibilidad. Apariencia de las instalaciones físicas, equipos, personal.

Fiabilidad. Habilidad para realizar el servicio prometido de manera confiable y cuidadosa. 
Capacidad de respuesta. Voluntad para ayudar a los usuarios.

Seguridad. Conocimiento del servicio prestado y habilidad para inspirar credibilidad y confianza.

Empatía. Atención individualizada al usuario.

Estas últimas dos dimensiones, seguridad y empatía, incluyen indicadores que resumen las dimensiones de comunicación, credibilidad, seguridad, competencia, cortesía, comprensión/conocimiento de los clientes y acceso, consideradas originalmente y excluidas durante el refinamiento de la escala de esta investigación (Carlos Ornelas, C. et al, 2010).

En consecuencia, el objetivo de este trabajo de investigación es: Delimitar los tipos de atributos tangibles o intangibles que el usuario prefiere dentro de un centro de análisis y transformación de información y contable (CTICD) para la diferenciación del servicio.

El artículo "Diagnóstico de la calidad del servicio de salas de cómputo para generar un plan de mejora en las salas de cómputo en un organismo académico", señala que las funciones de un centro de cómputo tienen "como fin principalmente dar un servicio de calidad a la comunidad estudiantil. Sin embargo, no se cumple adecuadamente con este fin, no satisfacen las necesidades de alumnado..." (Palma, Martínez, Álvarez y Estrada 2011).

Es importante señalar que "El mundo exige a las instituciones educativas de cualquiera que sea el sector, integrar dentro de sus procesos principales una dependencia a la tecnología, la misma que optimiza sus procesos y su administración, en el ámbito académico, las universidades integran del mismo modo este concepto." como lo menciona (Erazo Ayón, J.M. \& León R., W.,2018). "cuando se habla de la globalización de la ciencia puede argumentarse que se hace referencia a una serie de procesos equivalentes en las relaciones de ésta con la sociedad." Y la tecnología es un elemento básico para el manejo de este mundo globalizado. (Vesuri, 2014).
En un Laboratorio de Cómputo, se presentan problemas con características acumulativas situación que a su vez se refleja en la IES pública donde se desarrolla este trabajo de investigación, ya que un problema genera otro y a su vez genera otro y así sucesivamente, estos problemas no se documentan en su mayoría o no se llevan registros de ellos, y sólo el individuo que resuelve el problema, adquiere la experiencia y casi siempre no comparte los conocimientos adquiridos, arriesgando que si se vuelve a presentar la falla se perderá tiempo y esfuerzo en arreglarla si no es atendida por la persona que adquirió la experiencia pasada.

El Instituto Tecnológico Superior de Huatusco situado en el estado de Veracruz, es el lugar donde se ofrece la carrera de contador público este cuenta con una población de 421 alumnos en el ciclo escolar febrero- julio 2019.

Dentro de los laboratorios de Cómputo con los que cuenta la IES pública se desarrollan diversas actividades entre las que destacan:

\section{- Mantenimiento preventivo y correctivo de los equipos de cómputo y accesorios. Mantenimiento del mobiliario \\ - Atención a usuarios en préstamo de equipo y/o herramienta, solicitudes de impresión, renta de Internet, y soporte técnico \\ - $\quad$ Instalación de software con licencia.}

En lo sucesivo y para efectos de este trabajo se le denominará al laboratorio de cómputo escolar como Centro de Transformación de Información Digital y Contable (CTIDC) ya que este trabajo se limitará al estudio de aquellos laboratorios que están destinados al uso de los alumnos de la carrera de contador público.

Debido a la cantidad de usuarios que se atienden en los CTIDC, se presentan diversos problemas, la mayoría de ellos ocasionados por los usuarios, lo que origina que el personal del laboratorio se mantenga ocupado casi todo el tiempo y descuiden el aspecto administrativo y procedimental del laboratorio. Siendo esta la situación por la cual surge la siguiente hipótesis: Los atributos del servicio que se brinda al usuario, delimitan la existencia de un centro de análisis y transformación de información digital y contable. 


\section{Revisión de la Literatura}

El cambio tecnológico es una megatendencia significativa por derecho propio, que reconfigura constantemente economías y sociedades, en ocasiones de manera radical. Para el 2030, las empresas serán predominantemente digitalizadas, lo que permitirá que los procesos de diseño, fabricación y entrega sean altamente integrados y eficientes. Los costos de los equipos y la informática seguirán cayendo mientras que las tecnologías de reconocimiento de patrones, como los datos masivos y las máquinas que aprenden, mejorarán la capacidad para determinar las necesidades de los usuarios y la demanda general de la innovación. En conjunto, se están reorganizando una amplia variedad de mercados y esquemas laborales. El acceso a la educación y la adquisición de conocimiento y habilidades será una de las claves más importantes para mejorar las oportunidades de vida en México, la estrategia digital nacional en el país, la innovación y emprendimiento en economía digital, para mejorar la calidad de la educación con apoyo de las TICS (OCDE, 2016).

Visto de esta manera las instituciones escolares principalmente de educación superior están destinadas a contar con centros de cómputo donde el alumnado desarrolle las competencias necesarias para integrarse al campo productivo. Un centro de cómputo es una sala dedicada al equipo destinado al procesamiento de datos. La misma suele estar acondicionada para este tipo de circunstancia, debiendo contar con una determinada temperatura, medidas de seguridad y por supuesto, el hardware para su función principal (Editorial definición, 2014). En este trabajo un centro o laboratorio de cómputo escolar lo denominaremos como Centro de Transformación de Información Digital y Contable (CTIDC) el cual cuenta con diferentes atributos en el servicio los cuales lo caracterizan e influyen en su diferenciación.

\section{Atributos de un CTIDC}

Como señala (Alvares, Chaparro y Reyes, 2014) "debido a la acuciante necesidad de hacer mejoras en los Servicios Educativos, nace el interés de realizar trabajos de investigación que permitan analizar la satisfacción que tienen los Usuarios de las instituciones de Educación Superior (IES), asociando tal satisfacción con la calidad y competitividad de dichas instituciones".
Una gran cantidad de investigadores se han enfocado a abordar los atributos y/o indicadores de calidad en servicios. Ejemplo de ello son Mondo, T.S. con su propuesta TOURQUAL, en el sector turístico. (Mondo, T.S. et al,2016), basándose en "modelos ya existentes como el Groonros, Servperf, pero hizo mayor hincapié en ServQual "(Arias D., J.M. \& Reyes R., C.A., 2018). Servperf (Service Performance) propuesto por Cronin y Taylor (1992), citado por (Ibarra y Casas, 2014), "el cual recoge la percepción del cliente en el desempeño de la calidad del servicio otorgado". En otros estudios como el realizado por Motta y Barreto, 2017, marcan como objetivo de investigación "proponer un conjunto de atributos de servicio y de indicadores de rendimiento considerados relevantes desde la perspectiva de gerentes, profesionales y clientes para mejorar la competitividad de estas empresas".

En este trabajo los atributos intangibles que definen al servicio prestado en un CTIDC y que delimitan su existencia son:

La confiabilidad: habilidad para realizar un servicio prometido de manera cuidadosa y segura es decir la probabilidad de que todo funcione correctamente.

La atención al usuario y seguridad: Interacción entre los clientes y las organizaciones a través de múltiples canales de comunicación, es decir, que ambos tengan conocimiento del resultado del servicio prestado, así como de la habilidad para inspirar credibilidad y confianza en los usuarios.

La capacidad de respuesta y empatía: es dar de manera voluntaria con exactitud y perspicacia a los usuarios lo que necesitan de manera individualizada.

Sin embargo, la calidad del servicio depende en gran medida del desempeño de los empleados, donde según Bon (2008) un servicio es un medio para entregar valor a los clientes, facilitando los resultados que los clientes quieren conseguir sin asumir costos o riesgos específicos. 
Y la calidad del servicio según Pizzo (2013) es el hábito desarrollado y practicado por una organización para interpretar las necesidades y expectativas de sus clientes y ofrecerles, en consecuencia un servicio accesible, adecuado, ágil, flexible, apreciable, útil, oportuno, seguro y confiable, aún bajo situaciones imprevistas o ante errores, de tal manera que el cliente se sienta comprendido, atendido y servido personalmente, con dedicación y eficacia y sorprendido con mayor valor a lo esperado.

Por lo tanto, la percepción de satisfacción por parte del usuario, se relaciona con la calidad del producto o servicio, y ésta a su vez, con el cumplimiento de estándares o especificaciones del producto, lo cual se hace más difícil para las empresas de servicio debido a las características propias del sector, que es su intangibilidad (Chiriboga, Pérez, Hidalgo y Fuentes, 2018).

Estas características "específicas de los servicios, como la intangibilidad, la heterogeneidad y la simultaneidad, lo que dificulta su control y la valoración de sus resultados, para lo cual normalmente se emplean métodos indirectos, muchas veces por medio de la percepción de los clientes (Ruiz, 2006) citado por (Torres y Espinoza, 2017). La calidad del servicio percibida (CSP) por los estudiantes en la formación universitaria está recibiendo una creciente atención por parte de los investigadores, ya que abarcan diversas cuestiones "pero relacionadas, como son la determinación de las dimensiones que integran el constructo CSP, el diseño de modelos de gestión de calidad y los problemas asociados a su implantación, entre otras." (Martínez et al, 2013).

La calidad de acuerdo a Carrasco (2013) citado por (Arias 2018) señala que "La calidad de servicio es el principal factor de referencia para que las empresas promocionen la satisfacción de sus clientes y eleven, por tanto, sus niveles de competitividad. La calidad en el servicio consiste en igualar las expectativas de los clientes a sus percepciones cuando reciben el servicio". (p.17), que puede ser logrado a través de "incorporar valor mediante la conjunción de la calidad en sus procesos y la atención al cliente" (Chiriboga et al, 2018).
Otro autor como es el caso de Campoverde (2017) sumando estos conceptos de calidad y servicio al cliente, enfocar que "toda organización ya sea pública o privada debe orientar sus esfuerzos por cumplir con su promesa de valor, intentando brindar a sus clientes la atención que ellos esperan."

Como ya se mencionó existen diversos estudios que se han enfocado a abordar los atributos intangibles de los servicios dejando claro que la parte humanística a través de la interacción entre las personas, empleados y usuarios (parte ofertante y demandante) son las que crean escenarios de valor y diferenciación del servicio es decir, la interacciones y actuación entre los involucrados generan la satisfacción del usuario a través de un trato cálido, una relación de empatía y una forma de enfrentar y resolver problemas.

\section{Descripción del método}

Se realizó un estudio transversal y descriptivo (Borsotti, 2009). El estudio transversal analizó la existencia de un CTIDC por sus atributos tangibles e intangibles en el servicio que lo distingue y lo hace atractivo al usuario. Según Arias (1999, pág. 24) "La investigación descriptiva consiste en la caracterización de un hecho, fenómeno, individuo o grupo, con el fin de establecer su estructura o comportamiento". En este trabajo la investigación descriptiva presenta las preferencias de los usuarios de un CTIDC razón que delimita su existencia.

El trabajo se desarrolla en una IES pública (Instituto tecnológico Superior de Huatusco) la población de estudio incluyo 32 alumnos de los semestres segundo, cuarto y sexto de la carrera de contador público del ciclo escolar febrero- julio 2019.

Donde la frecuencia de uso del CTIDC: es del $56 \%$ de alumnos casi siempre, $32 \%$ de alumnos algunas veces y el $13 \%$ restante siempre (Gráfica 1). En consecuencia, se observa una frecuencia de uso de un CTIDC aceptable es decir de un $69 \%$ del alumnado. 
Frecuencia de uso de un CTIDC

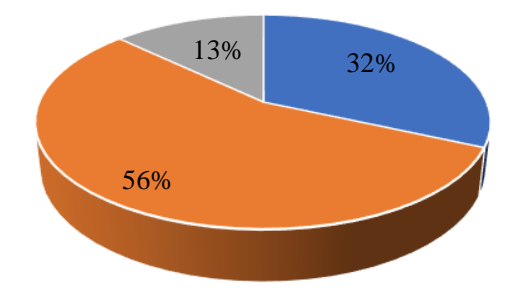

- Algunas veces " Casi siempre "Siempre

Gráfico 1 Frecuencia de uso de CTIDC

\section{Instrumentos de medición}

El instrumento de recolección de datos fue el cuestionario (Drew, Hardman y Hosp, 2007). El cuestionario determino la existencia de un CTIDC por sus atributos que el usuario considera de mayor influencia. El cuestionario evaluó los cambios en los ítems de atributos intangibles (servicio al cliente) y tangibles (infraestructura) que afectan la preferencia y aceptación del CTIDC.

El cuestionario utilizo la escala Likert. La escala Likert mide las opiniones y actitud de los encuestados (Pett, Lackey y Sullivan, 2003). Las respuestas son del 1 al 5, donde 1 no representa cambio alguno en la existencia de un CTIDC por el tipo de atributos tangibles 0 intangibles con los que cuenta y 5 siempre refleja cambios por cada cuestionamiento. La correlación de Pearson evalúa las preguntas. La evaluación verificó que los ítems no generan confusión por su redacción.

Con la finalidad de analizar la relación de dependencia ente las dimensiones de los atributos intangibles (variable explicativa) y la existencia de un CTIDC (variable de respuesta), se usó la prueba de independencia chi cuadrada (González, 2016). La prueba de chi cuadrada determina una asociación estadística entre dos atributos categóricos (Corder y Foreman 2009) El trabajo incluye el grafico del resultado de la chi cuadrada teórica de un par de variables.

\section{Resultados}

Validación del instrumento con correlación de Pearson.
La Tabla 1 presenta la correlación de Pearson. Esta correlación muestra que no existe repetición alguna entre las preguntas. Después de la validación, los 32 alumnos lo contestan.

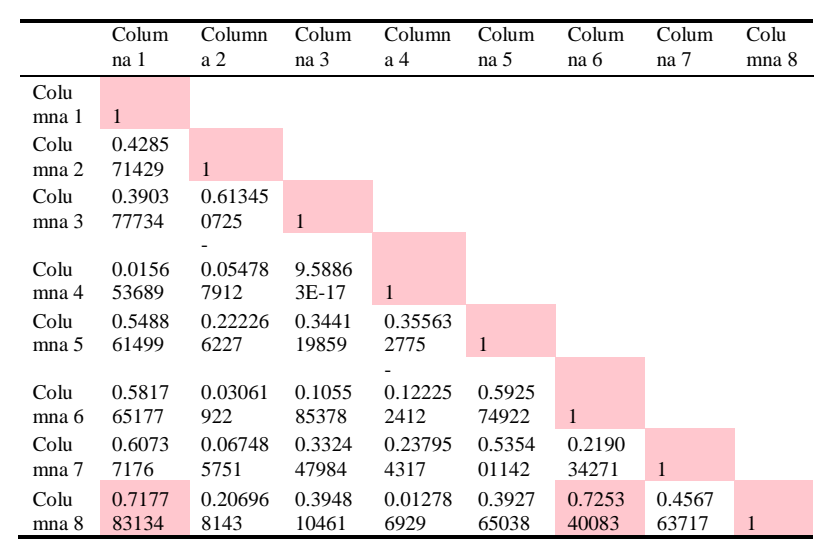

Tabla 1 Validación por correlación de Pearson

A continuación, se aplicó la prueba de chi cuadrada para hallar la relación de variables de dos en dos (representada por cada ítem). La tabla 2 muestra los resultados de los valores crudos tomados directamente de la encuesta. En la tabla 3 obtuvimos los valores esperados y en la tabla 4 se muestran los valores estadísticos de la chi cuadrada. En el caso específico que se muestra, se compararon las preguntas 1 y 3 .

Donde la 1 considera que es importante contar con un CTIDC.

Y la pregunta 3 detecta que los usuarios de los CTIDC toman en cuenta la atención amable y cálida que ofrece el personal que atiende un CTIDC (atributo intangible).

\begin{tabular}{|l|l|r|r|r|}
\multicolumn{2}{c}{} & \multicolumn{3}{c|}{ Existencia de CTIDC (1) } \\
\cline { 3 - 6 } \multicolumn{2}{c|}{} & si & no & totales \\
\hline \begin{tabular}{|l} 
Atención \\
amable \\
cálida (3)
\end{tabular} & yi & 30 & 1 & 31 \\
\cline { 2 - 6 } & no & 0 & 1 & 1 \\
\hline & totales & 30 & 2 & 32 \\
\cline { 2 - 6 }
\end{tabular}

Tabla 2 Tabla de contingencia, preguntas 1 y 3

\begin{tabular}{|c|c|c|c|c|}
\hline & & si & no & totales \\
\hline \multirow{3}{*}{\begin{tabular}{|l}
$\begin{array}{l}\text { Atención } \\
\text { amable } \\
\text { cálida (3) }\end{array}$ \\
\end{tabular}} & si & 29.0625 & 1.94 & 31 \\
\hline & no & 0.9375 & 0.06 & 1 \\
\hline & totales & 30 & 2 & 32 \\
\hline
\end{tabular}

Tabla 3 Tabla de valores esperados, preguntas 1 y 3 


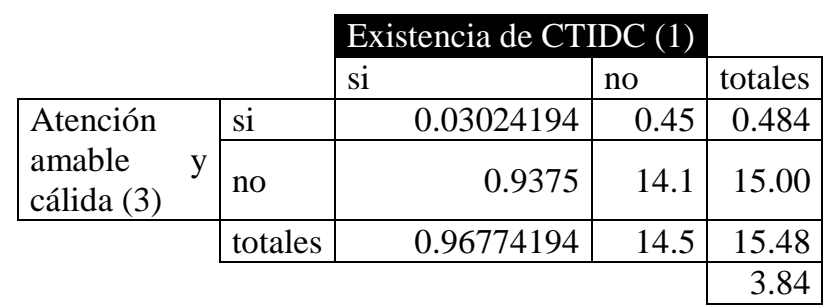

Tabla 4 Tabla de valores estadísticos, preguntas 1 y 3

El resultado de chi cuadrada observada o experimental arroja un valor de 15.48 (tabla 4); mientras que el valor de chi cuadrada teórica nos muestra un valor de 3.84. Se puede deducir entonces que las variables 11 y 3 son estadísticamente dependientes. En el Gráfico 2 se ejemplifica mejor esta dependencia. Podemos observar que la chi calculada está muy lejos de la chi teórica y fuera de la curva normal de Pearson para 1 grado de libertad. Se interpreta como un $\mathrm{p}$ valor casi de cero para la dependencia; 1 para la independencia.

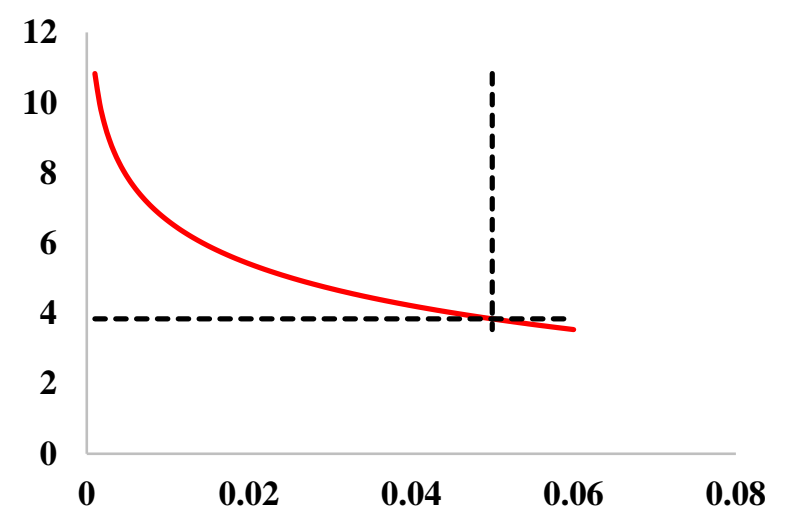

Gráfico 2 Gráfica de la función inversa teórica: p valor vs chi cuadrada de 1 grado de libertad. Se observa la intersección $(0.05,3.84)$ para chi teórica. 95\% de confianza para la independencia, 5\% de confianza para la dependencia

\section{Conclusiones}

El presente trabajo de investigación permite resaltar la importancia que tiene el servicio, la calidad de este, dentro de la caracterización de los atributos más relevantes para la medición de la misma en un centro de transformación de información digital y contable (CTICD), que es un espacio físico que cuenta con servicios de préstamo de equipos de cómputo, accesorios y/o herramientas digitales (software, soporte técnico, etc.), en una institución de educación superior pública en la zona centro del estado de Veracruz, México.
Lo cual cabe destacar dentro de la administración de las instituciones educativas y demás organismos que prestan servicio de este tipo, con la finalidad de prestar mayor atención en capacitar al personal que se encuentra al frente otorgando estos servicios y la calidad con la que se brinda, siendo el punto muchas veces de menor importancia al momento de programar y planificar mejoras de infraestructura académica, enfocándose primordialmente e incluso únicamente en la tecnología, por sobre todos los demás atributos.

El instrumento de recolección de datos fue validado mediante la correlación de Pearson, por lo que se considera como consistente y fiable como instrumento de medición, tomando en cuenta que la consistencia "se evalúa si los ítems que miden una misma dimensión presentan homogeneidad entre ellos" (Luján.Tangarife, J.A. et al, 2015). La fiabilidad de la escala es "el grado en que un instrumento es capaz de medir sin error" (Carrillo Sierra, M. et al, 2018).

Los estudiantes asociaron la calidad del servicio de la institución a dimensiones como confiabilidad, atención al usuario y seguridad, capacidad de respuesta y empatía; situación que valida la hipótesis planteada, estos atributos están relacionados con las instalaciones y equipamientos, además de otros factores. El nivel global de la calidad de los servicios se encuentra vinculadas a las percepciones y expectativas previas de los usuarios, así como a los atributos de las dimensiones planteadas. Por lo tanto, para satisfacer las expectativas de los usuarios, la administración de dichas instituciones debe enfocar sus esfuerzos en tomar como base los aspectos intangibles, como la atención al usuario, por sobre los aspectos tangibles y funcionales del servicio.

\section{Referencias}

Arias, F. (1999). El proyecto de Investigación. Guía para su elaboración (Vol.3) Caracas Venezuela; ORIAL.

Arias, J. y Reyes, R. (2018). Propuesta de intervención para medir los niveles de satisfacción de los visitantes que acuden a los atractivos turísticos de la zona urbana de Guayaquil. Tesis de licenciatura. Universidad Católica de Santiago de Guayaquil. Guayaquil, Ecuador. 
Álvarez Botello, Julio., Chaparro Salinas, Eva Martha., Reyes Pérez, Diana Elena., Estudio de la Satisfacción de los Estudiantes con los Servicios Educativos brindados por Instituciones de Educación Superior del Valle de Toluca. REICE. Revista Iberoamericana sobre Calidad, Eficacia y Cambio en Educación $</ i>$ [en línea]. 2015, 13(2), 5-26[fecha de Consulta 14 de septiembre de 2019]. ISSN. Disponible en: <a xmlns="http://www.w3.org/1999/xhtml"

target $=$ "_blank"

href="http://www.redalyc.org/articulo.oa?id=55 138743001">http://www.redalyc.org/articulo.o a?id=55138743001</a> Ralston, Anthony y Edwin D. Reilly (coordinadores), Encyclopedia of Computer Science 3rd Edition, Van Nostrand Reinhold, 1993 ISBN 0-442-27679-6, artículo «Digital Computers History».

Borsotti, C. (2009). Temas de metodología de la investigación. Madrid: Mino y Dávila.

Bon (2008), Gestión de Servicios de TI basada en ITII. V3. Primera Edición. Editorial del Gobierno Británico. Reino Unido.p.p.21

Campoverde Gonzaga, Diana Carolina (2017). Análisis de satisfacción de los estudiantes de grado de las Universidades privadas del Distrito Metropolitano de Quito, respecto a la atención recibida a nivel administrativo. Carrera de Ingeniería en Mercadotecnia. Universidad de las Fuerzas Armadas ESPE. Matriz Sangolquí. Ecuador.

Carlos Ornelas, C.; Montelongo Cortés, Y; Nájera Gallardo, Ma. La Calidad del Servicio de un centro de información. Conciencia Tecnológica, No.40, Julio. Diciembre, 2010., pp.5-9.

Carrillo Manuel Ulises. 1996. La Influencia de las tecnologías de la información en las organizaciones, ante la globalización un caso práctico, Tesis, UNAM, México, p.p. 29- 35.

Carrillo Sierra, Sandra Milena 1: Diego Andrés RIVERA Porras 2; Jesús Oreste FORGIONY Santos 3; Nidia Johanna BONILLA Cruz 4; María Luisa MONTANCHEZ Torres 5. (02/03/2018). Propiedades Psicométricas del cuestionario de Inclusión Educativa (CIE) en contextos escolares colombianos. ESPACIOS, 39, p.24.
Castells, M. (2001). La galaxia Internet. Reflexiones sobre Internet, empresa y sociedad. Editorial: Plaza y Janés, Madrid, España.

Casas de la Torre, F. y Suárez. Bertha , Trabajo presentado en el congreso internacional de investigación e innovación 2018, por. Experiencia de mejora en un centro de cómputo aplicando principios administrativos. pág. 848.

Centro de Cómputo. Sitio: Definición MX. Fecha: 20/06/2014. Autor: Editorial Definición MX.

URL:https://definicion.mx/centro-de-computo/. Lugar: Ciudad de México.

Chiriboga C., E.; Pérez Z, M.A.; Hidalgo L., J.A.; Fuentes T., A. (2018) Modelo de gestión GAP y la maximización en el nivel de satisfacción del cliente: caso restaurante Casa Blanca. Revista Científica ECOCIENCIA. ISSN: 1390-93, Edición especial, diciembre 2018. Guayaquil, Ecuador.

Corder G.W.,Foreman $\quad$ D., I. 2009: Nonparameteic Statistics for Non Satatiscians: A step-bystep Approach. Hoboken, New Jersey. Wiley.

Drew, Clifford J- Hardman, Michal L.- Hosp, John L. (2007). Design \& Conducting Research in Education.

Erazo Ayón, José Miguel \& León Romero,Wendy Vanessa.(2018). Análisis, diseño e implementación de un modelo de gestión basado en un proceso crítico del Centro de cómputo de la Universidad Católica Santiago de Guayaquil. http://repositorio.ucsg.edu.ec/handle/3317/1080 0 .

Gordon, Laing (2004). Digital Retro: The Evolution and Design of the Personal Computer. Sybex. p. 192. ISBN 978-0-7821-4330-0.

Hebe Vessuri. "Cambios en las ciencias ante el impacto de la globalización". Revista de Estudios Sociales, n.o 50 (2014): 167173. https://doi.org/10.7440/res50.2014.16.

Ibarra M., L.E. y Casa M., E.V. (2014). Aplicación del modelo Servperf en los centros de atención Telcel, Hermosillo: una medición de la calidad en el servicio. Universidad Estatal de Sonora. Contaduría y Administración 60 (1), enero-marzo 2015: 229-260. México.

GÓMEZ-MÁRQUEZ, Montserrat, RUIZ-HERNÁNDEZ, Ana Lilia, GONZÁLEZ-SÓBAL, Martín y ROSAS-BALTAZAR, Miguel Eduardo. Identificación de los atributos del servicio en un centro de transformación de información digital y contable. Revista de Tecnologías de la Información. 2019 
Luján-Tangarife, J.A. Cardona-Arias, J.A. (2015). CONSTRUCCIÓN Y VALIDACIÓN DE ESCALAS DE MEDICIÓN EN SALUD: revisión de propiedades psicométricas. iMedPub Journals, 11, pp.1-10.

Martínez-Argüelles, María Jesús; BLANCO, Miguel; CASTÁN, José M. (2013). «Las dimensiones de la calidad del servicio percibida en entornos virtuales de formación superior» [artículo en línea]. Revista de Universidad y Sociedad del Conocimiento (RUSC). Vol. 10, n. ${ }^{\circ}$ 1, págs. 89-106. UOC. [Fecha de consulta: 14/09/19]. ISSN 1698-580X.

Motta, Elis María, and Mario Cesar Barreto Moraes. "A proposal of service attributes and performance indicators for fitness centers/proposta de atributos de servicos e de indicadores de desempenho para academias fitness/propuesta de atributos de servicios y de indicadores de rendimiento para los gimnasios." Podium: Sport, Leisure and Tourism Review, vol. 6, no. 1, 2017, p. 124+. Gale Academic Onefile, Accessed 14 Sept. 2019.

OECD (2016), OECD Science, Technology and Innovation Outlook 2016, OECD Publishing, París, http://dx.doi.org/10.1787/sti_in_outlook2016-en. 2018 Microsoft Latín América para esta edición en español

Palma L., R. Martínez A., M.\& Álvarez B., J. \& Estrada G., C. Diagnóstico de la calidad del servicio de salas de cómputo para generar un plan de mejora en las salas de cómputo en un organismo académico. $9^{\circ}$. Congreso Internacional en Competitividad Organizacional. RILCO, red de investigación Latinoamericana en competitividad de organizaciones. 10,11 y 12 de agosto del 2011.

Pett, M.A., Lackey, N.R., \& Sullivan, J.J. (2003) Making sense of factor análisis: The use of factor análisis for instrument developed in health care research. Sage.

Pizzo, M. (2013). Construyendo una definición de calidad en el servicio, recuperado el 20 de agosto 2013, de la fuente: http://comoservirconexcelencia.com/blog/construyendouna-definición-de-calidad-en-el-servicio/.html
Quiroz, D. L. Z., \& Quiroz, M. S. Z. (2019). Las tecnologías de la información y las comunicaciones (TICS) en la educación superior: consideraciones teóricas. refcale: Revista Electrónica Formación y Calidad Educativa. ISSN 1390-9010, 7(1), 213-228.

Ramos, B. (2019). Percepción de la calidad de servicio entre los colaboradores en la I.E.E. Harvard empleando la escala SERVQUAL. Tesis para optar el título de Licenciada en Administración, Escuela Académico Profesional de Administración, Universidad Continental, Huancayo, Perú.

Sistema de gestión documentaria, contable y académica para el centro de cómputo e informática de la Universidad Privada San Carlos - 2014, Palomino Onofre, Sindy Catelin URI:

http://repositorio.upsc.edu.pe/handle/UPSC/438 3 Fecha: 2013-12-20

Savi Mondo, Tiago., Gonçalves Silveira Fiates, Gabriela., ATRIBUTOS DE LA CALIDAD DE SERVICIOS EN ATRACTIVOS TURÍSTICOS. Un estudio netnográfico en el uso del protocolo TOURQUAL.http://www.w3.org/1999/xhtml"> Estudios y Perspectivas en Turismo $</ \mathrm{i}>$ [en linea]. 2016, 25(2), 124-144[fecha de Consulta 14 de septiembre de 2019]. ISSN: 0327-5841. Disponible en: <a xmlns="http://www.w3.org/1999/xhtml" target="_blank"ref="http://www.redalyc.org/art iculo.oa?id=180744490002">http://www.redaly c.org/articulo.oa?id=180744490002</a .

Suarez Baltazar, Raúl. (2015). El nivel de calidad de servicio de un centro de idiomas aplicando el modelo Servqual caso: Centro de idiomas de la Universidad Nacional del Callao periodo 2011-2012. Universidad Nacional Mayor de San Marcos. Facultad de Ciencias Administrativas. Unidad de Posgrado. Lima, Perú.

Torres F., J. y Espinoza L., I. (2017). Evaluación de la percepción de la calidad de los servicios bancarios mediante el modelo SERVPERF. Universidad del Istmo, México. Contaduría y Administración 62 (2017) 1270-1293. www.sciencedirect.com. 
Tumino, Marisa Cecilia., Poitevin, Evelyn Ruth., Evaluación de la calidad de servicio universitario desde la percepción de estudiantes y docentes: caso de estudio. <i xmlns="http://www.w3.org/1999/xhtml">REIC E. Revista Iberoamericana sobre Calidad, Eficacia y Cambio en Educación $</ \mathrm{i}>$ [en linea]. 2014, 12(2), 63-84[fecha de Consulta 14 de septiembre de 2019]. ISSN. Disponible en: <a xmlns="http://www.w3.org/1999/xhtml"

target="_blank"

href="http://www.redalyc.org/articulo.oa?id=55 130462004">http://www.redalyc.org/articulo.o a? id $=55130462004</ a>$

Usman, S., Mehmood, R., y Katib, I. (2018). Big data and hpc convergence: The cutting edge and outlook. Lecture Notes of the Institute for Computer Sciences, Social Informatics and Telecommunications Engineering Smart Societies, Infrastructure, Technologies and Applications, 11-26. doi: 10.1007/978-3-31994180-6\4 